\title{
Cosmic star formation rate density from DLAs and the HI-metallicity relation
}

\author{
J.L. Hou \\ Shanghai Astronomical Observatory, Shanghai, 200030, China \\ email: hjlyx@shao.ac.cn
}

\begin{abstract}
Some basic observational properties of Damped Lymna $\alpha$ systems (DLAs) have been examined by Monte Carlo simulation based on the assumption that DLAs are disks form at the center of dark halo at redshift 3 . We found that the predicted cosmic star formation rate density contributed by DLAs is consistent with the most recent observations if the star formation timescale in DLAs is assumed to be $1 \sim 3$ Gyr. By comparing the UV luminosity function between DLA host galaxies and that of Lyman Break Galaxies(LBGs), we show that the DLAs host galaxies are much fainter than LBGs, and that only few percent of DLAs can host LBGs. The discrepancy between model prediction and observation in the correlation between metallicity and HI column density for DLAs is reproduced on the basis of Kennicutt star formation formalism at high redshift. It is quite difficult to interpret this concerning the complexities of dust depletion, star formation mode as well as model limitations. Further investigations are needed.
\end{abstract}

Keywords. Galaxies: formation and evolution, quasars: absorptions, galaxies: DLAs

\section{Introduction}

Among various observed properties of DLAs, there exists a very unusual property, that is there seems to be a trend of anti-correlation between observed $[\mathrm{Zn} / \mathrm{H}]$ and $\mathrm{HI}$ column densities, which is independent of redshift (see details in Hou et al. 2001).

DLAs are observed at different redshift, so they are good tracers to reconstruct the cosmic star formation history (Hopkins et al. 2005). An interesting question is how much can DLAs contribute to the cosmic star formation rate density and how it is related to the LBGs (Wolfe et al. 2003).

In this contribution, we report our model results of DLAs based on the Semi-Analytical Model (SAM) in the context of standard hierarchical picture by assuming that DLAs are hosted by disk galaxies. Details can be found in Hou et al. (2005).

\section{The model}

The SAM of galaxy formation comes from that for disk galaxies suggested by Mo, Mao \& White (1998). Details can be found in Mo et al. (1998) and Hou et al. (2005). We use Monte Carlo simulation to generate the model DLAs by applying a random sight line passing through the disk. DLAs galaxies are selected by applying star formation and chemical evolution in the disk.

\section{Cosmic star formation rate density from DLAs host galaxies}

Our model is able to predict a number of DLAs properties, such as the metallicity distribution, the frequency distribution of HI column density, number density, star formation rate density contributed by DLAs. We found that all those quantities are 
quite comparable to the observed properties. Especially, based on the selected DLA sample, we get the predicted cosmic SFR density contributed by DLAs at $z \sim 3$ about $\log (\mathrm{SFR}) \sim-0.75\left(h M_{\odot} y r^{-1} M p c^{-3}\right)$, which is quite consistent with most recent observations of Wolfe et al. (2003). The SFR densities resulted from DLAs are similar to that from high redshift luminous galaxies, which are observed as Lyman Break Galaxies (LBGs). By calculating the luminosity functions of selected DLA host galaxies, we found that the typical $R_{\mathrm{AB}}$ magnitude of the predicted DLA hosts is $\sim 30$, which is much fainter than LBGs with typical $R_{\mathrm{AB}} \sim 25$. This implies that a typical DLA host galaxy in our model has its SFR 100 times smaller than a typical LBG. Because the number density of DLA host galaxies is about 0.26 (Hou et al. 2005) which is about 100 times larger than the observed comoving number density of LBGs, our predictions of cosmic SFR density contributed by DLAs is similar to that of LBGs at $z \sim 3$.

\section{The anti-correlation between $\log \left(N_{H I}\right)$ and metallicity}

We examined the correlation between metallicity and HI column density for the selected DLA sample. As expected, model predictions show an opposite trend compared with observations. Boissé et al. (1998) suggested that the absence of DLAs with low metallicities and low HI column densities could be attributed to the observational selection effects. And, the lack of DLAs with both high metallicities and column densities is due to the dust obscuration (see also Prantzos \& Boissier 2000; Vladilo \& Peroux 2005).

Ellison et al. $(2001,2004)$ have surveyed a sample of DLAs towards radio selected quasars and found no significant difference from the optical survey in the HI distribution of DLAs. This implies that dust obscuration may play a relatively minor role (see also Prochaska \& Wolfe 2002). Schaye (2001) has proposed a physical explanation for the upper limit of HI column density in DLAs by assuming that clouds with $N_{\mathrm{HI}}>10^{22} \mathrm{~cm}^{-2}$ will not appear since $\mathrm{HI}$ will be converted into $\mathrm{H}_{2}$ before reaching such high column density. Survey for molecular clouds in high redshift DLAs may help us for further understanding. Another possibility could be the inadequacy of the adopted Schmidt type Kennicutt star formation prescription (Kennicutt 1998) at high redshift. At least some clues are available from observations of Wolfe et al. (2003). Therefore, it is still unclear what is the underlying physics for the observed HI-metallicity anti-correlation of DLAs.

\section{Acknowledgements}

This work is supported by NSFC10173017, 10133020, NKBRSF 1999075404.

\section{References}

Boissé P., Le Brun V., Bergeron J. \& Deharveng J.M. 1998, A\&A A, 333, 841

Ellison S., Yan L., Hook I., Pettini M., Wall J. \& Shaver P. 2001, A\&A, 379, 393

Ellison S.L., Churchill C.W., Rix S.A. \& Pettini M. 2004, ApJ, 615, 118

Hopkins A.M., Rao S.M., \& Turnshek D.A. 2005, ApJ, submitted, astro-ph/0505418

Hou J.L., Boissier S. \& Prantzos N. 2001, A\& A, 370, 23

Hou J.L., Shu C.G., Shen S.Y., Chang R.X., Chen W.P. \& Fu C.Q. 2005, ApJ, 624, 561

Kennicutt R. 1998, ApJ, 498, 541

Mo H.J., Mao S.D. \& White S.D.M. 1998, MNRAS, 295, 319

Prantzos N. \& Boissier S. 2000, MNRAS, 315, 82

Prochaska J.X. \& Wolfe A.M. 2002, ApJ, 566, 68

Schaye J. 2001, ApJ, 562, L95

Vladilo G \& Peroux C. 2005, A\&AA, submitted, astro-ph/0502137

Wolfe A.M., Gawsier E. \& Prochaska J.X. 2003, ApJ, 593, 235 